\title{
ASSESSMENT OF WALL-THINNING IN CARBON STEEL PIPE BY USING LASER-GENERATED GUIDED WAVE
}

\author{
DOYOUN KIM, YOUNHO CHO* and JOONHYUN LEE \\ Graduate School of Mechanical Engineering, Pusan National University \\ Jangjeon-dong, Gumjeong-gu, Busan, 609-735, Korea \\ "Corresponding author. E-mail : mechcyh@pusan.ac.kr \\ Received December 15, 2009 \\ Accepted for Publication July 13, 2010
}

The objective of this research is to estimate the crack location and size of a carbon steel pipe by using a laser ultrasound guided wave for the wall thinning evaluation of an elbow. The wall thinning of the carbon steel pipe is one of the most serious problems in nuclear power plants, especially the wall thinning of the carbon steel elbow caused by Flow-Accelerated Corrosion (FAC). Therefore, a non-destructive inspection method of elbow is essential for the nuclear power plants to operate safely. The specimens used in this study were carbon steel elbows, which represented the main elements of real nuclear power plants. The shape of the wall thinning was an oval with a width of $120 \mathrm{~mm}$, a length of $80 \mathrm{~mm}$, and a depth of $5 \mathrm{~mm}$. The $\mathrm{L}(0,1)$ and $\mathrm{L}(0,2) \mathrm{modes}$ variation of the ultrasound guided wave signal is obtained from the response of the laser generation/air-coupled detection ultrasonic hybrid system represent the characteristics of the defect. The trends of these characteristics and signal processing were used to estimate the size and location of wall thinning.

KEYWORDS : Ultrasound Guided Wave, Wall-Thinning, Air-Coupled Transducer, Flow-Accelerated Corrosion

\section{INTRODUCTION}

Carbon steel is one of the principal structural materials in power plants. Since local wall thinning caused by FAC occurs inside the elbows due to the flowing high temperature and high pressure water with high velocity, a big disaster may occur if this defect continues to grow without stopping. Therefore, structural evaluation of elbows with local wall thinning becomes more important in order to maintain the integrity of coolant piping systems [1, 2].

Most of the inspections were carried out using point by point examination. This method is inefficient and it also takes a lot of time to inspect large area structures. In these situations, the developed method, which inspects structures using guided waves, is able to overcome the drawbacks mentioned above compared to the conventional technique.

The ultrasonic guided wave technique is one of the powerful tools that can detect reduction and has lots of benefits over conventional ultrasonic methods due to such features as lower cost, ease of operation, and testing speed. Moreover, broad-band and multi-mode guided waves, such as those generated by a laser system, have the potential to detect flaws in various sizes [3-5]. In this study, laser generation/ air-coupled transducer ultrasonic hybrid systems were employed as a way to detect the defect of elbow by using guided wave that passed through the elliptical defeat of the elbow. In addition, a linear slit array was used to direct the laser-generated guided wave and determine the wavelength. An air-coupled transducer was used as a guided waves detector, which was able to detect specific modes among guided waves by adjusting its receiving angle to the direction of the leak from the selected mode.

\section{SELECTIVE GENERATION AND RECEPTION OF GUIDED WAVE MODE}

The problem in laser based guided wave testing is the difficulty in generating a desired mode due to the dispersive nature of Lamb waves [6]. While the excitation of a particular mode is made by a laser pulse, the different components of the wave travel indifferent speeds, and at least two modes are present, even at a low frequency range. This could make the evaluation of defects difficult due to interpretation of the received signal.

In this study, the selective generation and reception of guided wave modes were achieved by using a technique that also used the relation of dispersion curves and linear slit array [7]. Figure 1(a) shows the process of selective generation using this linear slit array. The elements gap $(\Delta s)$ shown in Figure 1(a) is equal to the wavelength of generated modes and is illustrated as a diagonal line with a slope of $\Delta s / d$ in Figure 1(a). 
The active modes lie on intersection points between the line and phase velocity of dispersion curves. Therefore, it is possible to generate specific modes selectively by adjusting the elements gap. The method to receive the modes generated by the above-mentioned technique is to rotate the air-coupled transducer by the angle based on Snell's law for the propagation velocity in air $\left(\mathrm{C}_{\text {air }}\right)$ and the phase velocity of

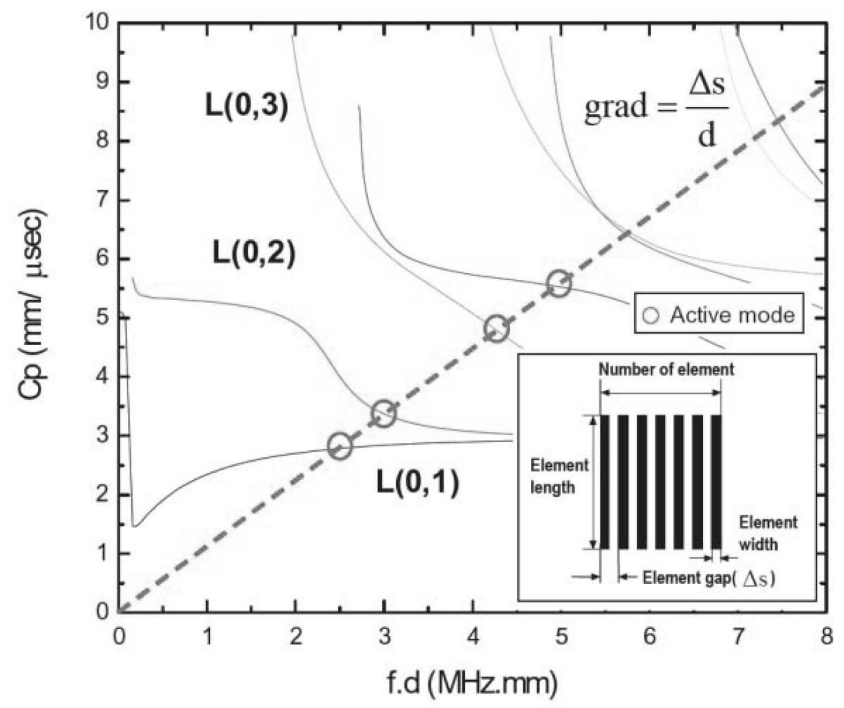

(a) Phase velocity of selected mode in dispersion curve

\section{Snell's law}

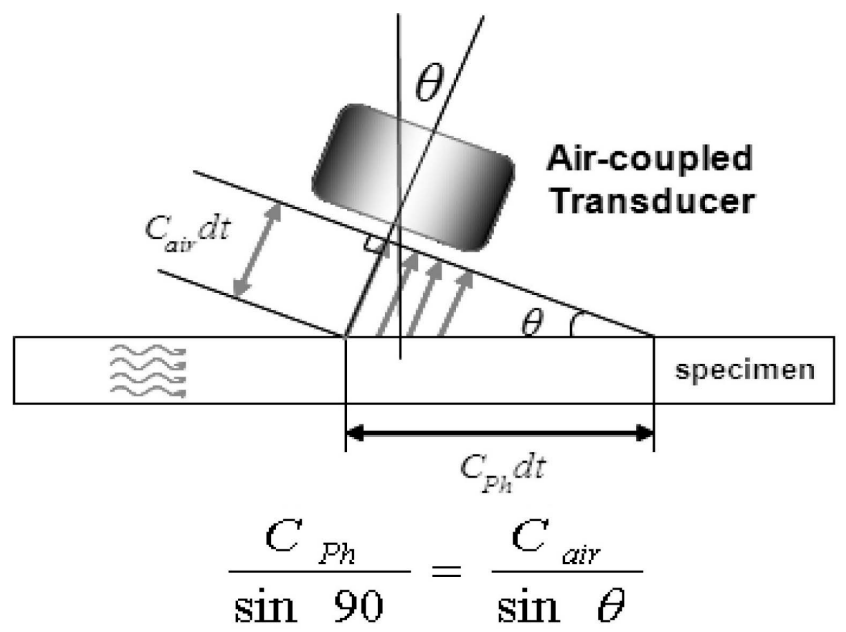

(b) Determination of receipt angle for specific mode

Fig. 1. Optional Receipt of Guided Wave Mode the specific mode (Cp), as shown in Figure 1(b). In this study, the velocity of waves in the air was $340 \mathrm{~m} / \mathrm{s}$ and the phase velocity of modes was obtained, as shown in Figure 1(a).

This study adopted the modes of $\mathrm{L}(0,1)$ and $\mathrm{L}(0,2)$ as the suitable modes for the experiment due to being readily excited and received experimentally at a low frequencythickness and being only slightly dispersive [8]. Table 1 shows the frequencies, phase velocities, and reception angles of $\mathrm{L}(0,1)$ and $\mathrm{L}(0,2)$ modes at $8 \mathrm{~mm}$ slit spacing.

Table 1. Theoretical Values of $L(0,1)$ and $L(0,2)$ Modes at $8 \mathrm{~mm}$ Wavelength on Each Defect

\begin{tabular}{c|c|c|c|c}
\hline $\begin{array}{c}\text { Wavelength } \\
{[\mathrm{mm}]}\end{array}$ & Mode & $\begin{array}{c}\text { Frequency } \\
{[\mathrm{kHz}]}\end{array}$ & $\begin{array}{c}\text { Phase } \\
\text { velocity } \\
{[\mathrm{mm} / \mu \mathrm{sec}]}\end{array}$ & $\begin{array}{c}\text { Receiving } \\
\text { angle }\left[\theta^{\circ}\right]\end{array}$ \\
\hline \multirow{2}{*}{$8 \mathrm{~mm}$} & $\mathrm{~L}(0,1)$ & 305 & 3.2 & 6.09 \\
\cline { 2 - 5 } & $\mathrm{L}(0,2)$ & 382 & 3.6 & 5.4 \\
\hline
\end{tabular}

\section{SPECIMEN AND EXPERIMENTAL SETUP}

The specimen used in the test was an $8.5 \mathrm{~mm}$ thick carbon steel elbow. The differences in ultrasonic wave propagating in the defect region and defect-free region were studied in order to evaluate the guided wave interaction with a defect in the elbow. An elliptical defect with a constant width of $120 \mathrm{~mm}$ and a depth of $5 \mathrm{~mm}$ was machined on the inner surface of an $8.5 \mathrm{~mm}$ thick elbow that had a diameter of $218 \mathrm{~mm}$. Figure 2 shows the shape of the side and front mentioned defect on the carbon steel elbow.

A schematic diagram of the apparatus used to perform the experiment is shown in Figure 3. As shown in this figure, the laser and air-coupled transducer were positioned on the same side of the test elbows and acted as the generator and

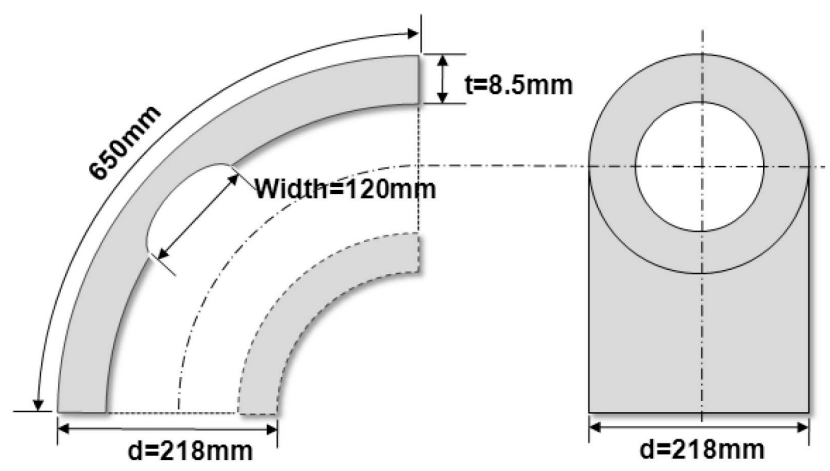

Fig. 2. Shape of Defects in $8.5 \mathrm{~mm}$ Thick Elbow 


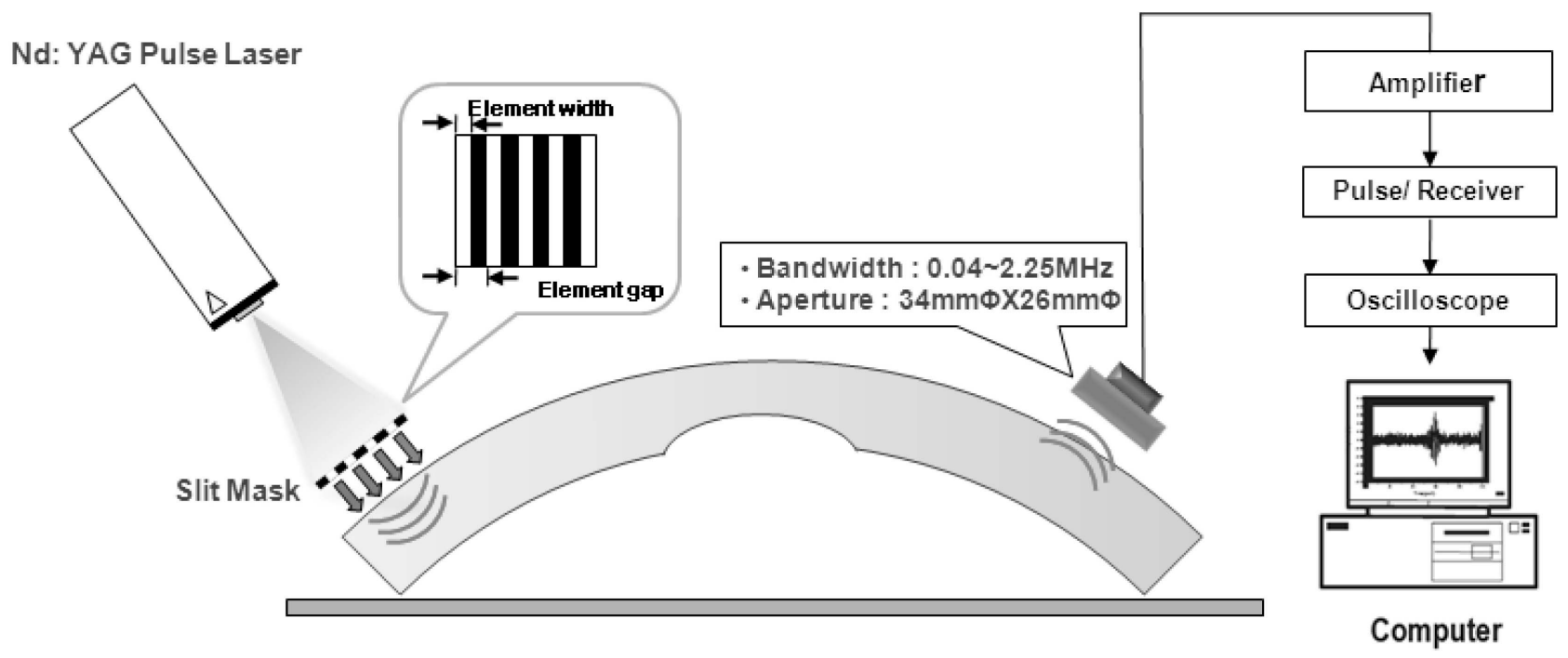

Fig. 3. Schematic Diagram of Experimental Setup

detector of the guided wave signal scanning in steps of $10 \mathrm{~mm}$ steps along the longitudinal direction. The wavelength of the fiberized Nd:YAG pulse laser system, which was used to generate waves were $532 \mathrm{~nm}$ and the emitted energy of this pulse laser system was $32 \mathrm{~mJ}$ at one pulse. The beam of this laser illuminated a linear array slit while the transmitted beam acted as a line source on the elbow. The guided wave generated by this source propagated a separation distance to start at $160 \mathrm{~mm}$ and end at $380 \mathrm{~mm}$ between the source to the receiver, which was perpendicular to the surface of the elbow. This was subsequently detected using an air coupled transducer with a standoff from $5 \mathrm{~mm}$ the outer surface of the elbow. In addition, the received signals from the aircoupled transducer were magnified by the amplifier and displayed through the signal averaging scheme with 1000 sampling data on the screen of the oscilloscope. Here, the interval between the slits, the width, and the number of slits were found to be $8 \mathrm{~mm}, 4 \mathrm{~mm}$, and 7 respectively.

\section{EXPERIMENTAL RESULTS}

\subsection{Characteristic of $L(0,1), L(0,2)$ Modes in Defect Region of Elbow}

Figure. 4 shows the variation of amplitude in the defect region. After scanning in a right to left direction, as shown in Figure 4, it became possible to evaluate the defects by varying the $\mathrm{L}(0,1)$ and $\mathrm{L}(0,2)$ modes. The signal of the $\mathrm{L}(0,1)$ mode appeared to range from $16 \mathrm{~cm}$ to $40 \mathrm{~cm}$, regardless of the defect region, but the amplitude of the $\mathrm{L}(0,2)$ mode disappeared from $23 \mathrm{~cm}$ to $33 \mathrm{~cm}$, which started the defect region. This result indicates that the signal on the defect region was affected by the defect.

Figure 5 shows the characteristics of the frequency spectra of an $\mathrm{L}(0,1)$ mode with a frequency of $381 \mathrm{kHz}$ (theoretical value is $376 \mathrm{kHz})$ and an $\mathrm{L}(0,2)$ mode with a frequency of $434 \mathrm{kHz}$ (theoretical 1 value is $423 \mathrm{kHz}$ ). The plots in Figures 5 (a)-(b) are the frequency spectra of these modes in the $0 \sim 1 \mathrm{MHz}$ range. The frequency spectra were obtained by performing a Fast-Fourier Transform (FFT) of the timedomain waveforms, with the magnitude of the $\mathrm{L}(0,1)$ mode that has a center frequency of $381 \mathrm{kHz}$ in the defected region being increased by $13 \%$. However, the $\mathrm{L}(0,2)$ mode of waves propagating in the defected region suffered a dramatic attenuation. The maximum decrease in the center peak magnitude of the signal with a frequency of $434 \mathrm{kHz}$ was $81 \%$. This is because as the fd changed from the dispersion curve, the $\mathrm{Cp}$ and $\mathrm{Cg}$ of the $\mathrm{L}(0,1)$ mode did not change too much, but one of the $\mathrm{L}(0,2)$ modes changed a lot, which meant that the $\mathrm{L}(0,2)$ mode was more sensitive than the $\mathrm{L}(0,1)$ mode. A simple introduction of the dispersion curve of the two modes could be added in the future.

\subsection{Defect Localization along a Longitudinal Direction}

As shown in Figure3, which shows the experimental setup, guided waves were received with a constant source/receiver separation along the longitudinal direction. The air-coupled transducer passed by the center of defect, which obtained clear characteristics of a defect. Figure 6 shows the results from a line scan in the right and left direction, where there was synthesis of right and left maximum magnitudes by 


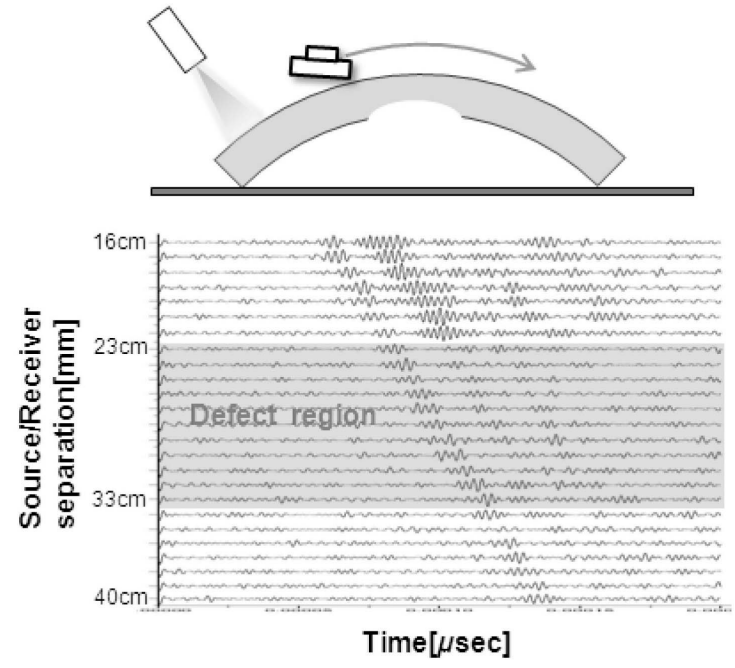

(a) Scanning to right direction

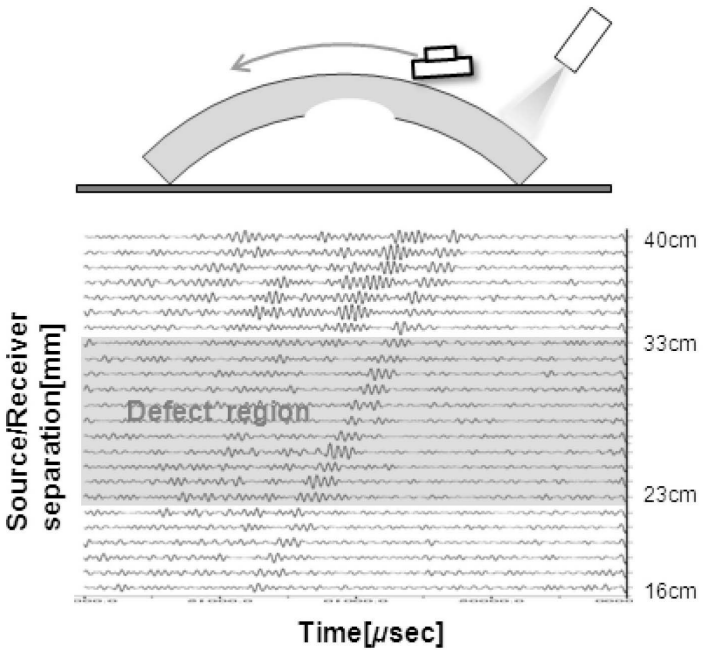

(b) Scanning to left direction

Fig. 4. Guided Wave Signals of L(0,1), L(0,2) Mode in Elbow with Defect region

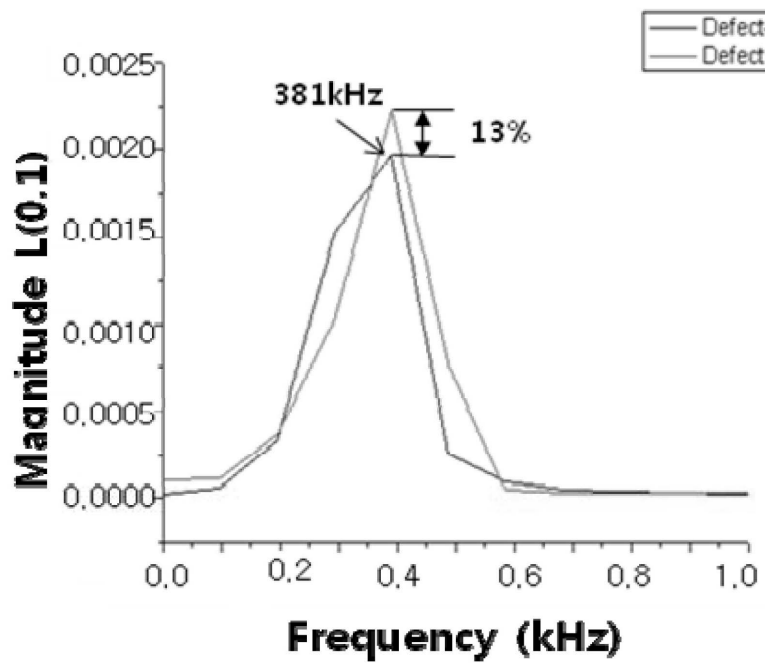

(a) Frequency spectrum of $\mathrm{L}(0,1)$

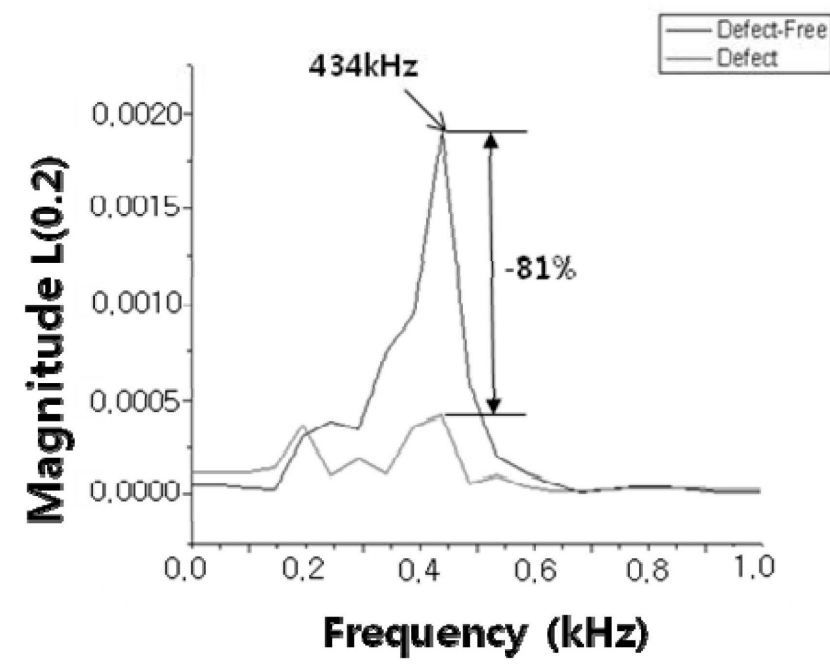

(b) Frequency spectrum of $\mathrm{L}(0,2)$

Fig. 5. Signal Characteristics of L $(0,1)$ and L $(0,2)$ Mode on Frequency Spectrum

using the pitch-catch method in a defect region. The maximum magnitude of the frequency spectrum in $\mathrm{L}(0,1)$ and $\mathrm{L}(0,2)$ modes were plotted as a function of the scan position by scanning in steps of $10 \mathrm{~mm}$ along the longitudinal direction respectively.
Figure 6(a)-(b) show that the magnitude of the $\mathrm{L}(0,1)$ mode increased linearly, but it is not clearly distinguished from the starting point of the defect region as the depth of the elliptical defect increased. However, the maximum magnitude of the $\mathrm{L}(0,2)$ mode decreased rapidly in the 


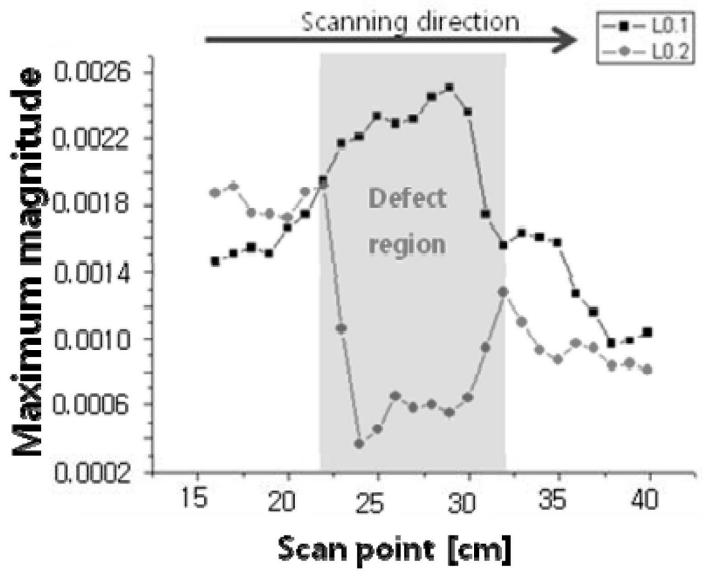

(a) Magnitude of right direction

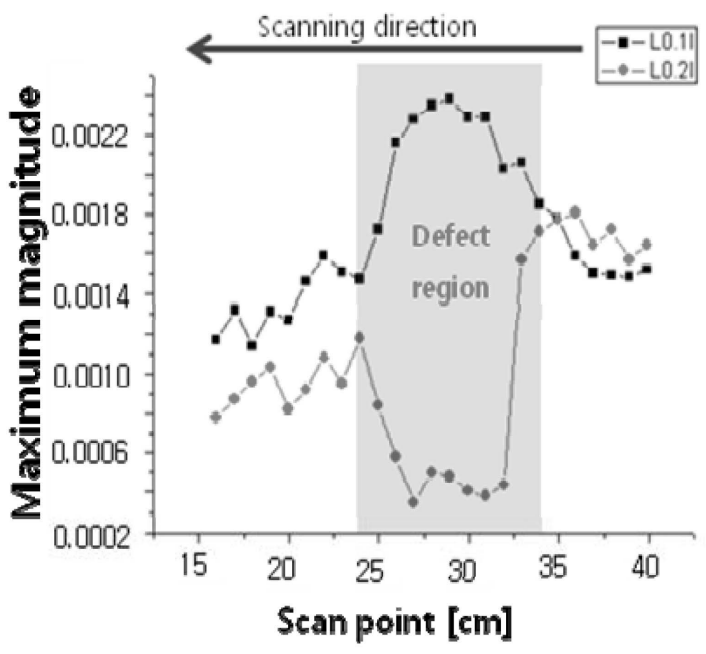

(b) Magnitude of left direction

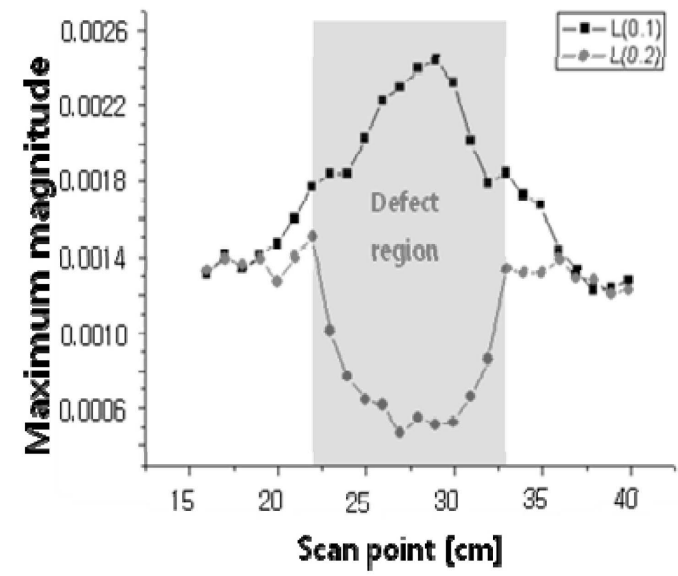

(c) Synthesis of right and left maximum magnitudes

Fig. 6. Maximum Magnitude Using Line Scan Technique on Defect Region defect region.

Figure 6(c) is synthesized from the right and left maximum magnitude. The $\mathrm{L}(0,1), \mathrm{L}(0,2)$ modes show the inverse characteristics of the defect region, Specifically, the $\mathrm{L}(0,2)$ mode indicates a more accurate relation between the depth of defect and variation of signal. As a result of the maximum magnitude, a relative depth of elliptical defect is estimated.

\section{CONCLUSIONS}

The possibility for estimating thickness reduction using the group velocity of guided waves was applied to the elbow. As a result, in order to evaluate the thickness reduction using the group velocity, mode identification was conducted by time-frequency analysis. In the elbow, $\mathrm{L}(0,1)$ and $\mathrm{L}(0,2)$ modes appeared in the defect-free region, but the amplitude of the $\mathrm{L}(0,2)$ mode disappeared in wall-thinning. The characteristics of the maximum magnitude of the frequency spectrum of the $\mathrm{L}(0,2)$ mode was varied in the defect region. Therefore, we believe that it is possible to evaluate the wall-thinning of the elbow by using the ratio of $\mathrm{L}(0,2)$ to $\mathrm{L}(0,1)$ to measure the magnitude quantitatively.

\section{ACKNOWLEDGMENTS}

This research was supported by the World Class University (WCU) program through the National Research Foundation of Korea funded by the Ministry of Education, Science and Technology (R33-10155) and BK21(Brain Korea 21) program. The authors would like to express their gratitude for their financial support, which made this research possible. The authors are also deeply grateful to Professor J. D. Achenbach from Northwestern University for the invaluable comments and technical advice he provided on this work via the WCU program.

\section{REFERENCES}

[1] R.B. Dooley, V.K. Chexal: Flow-accelerated corrosion of pressure vessels in fossil plants, International Journal of Pressure Vessels and Piping, Vol. 77(2000), p. 85-90.

[2] A. Demma, P. Cawley, M. Lowe, A.G. Roosenbrand, B. Pavlakovic: The relection of guided waves form notches in pipes: a guide for interpreting corrosion measurements, NDT\&E International, vol. 37(2004), p. 167-180

[ 3 ] M.J.S. Lowe, D.N. Alleyne, P. Cawely: Defect detection in pipes using guided waves, Ultrasonics, Vol. 36(1996), p. 147-154.

[4] Kyungyoung Jhang, Hongjoon Kim, Hyunmook Kim and Job Ha: Laser Generation of Focused Lamb Waves, Journal of the Korean Society for Nondestructive Testing, Vol. 22, No. 6(2002), p. 637-642

[ 5 ] W.M.D. Wright, D.A. Hutchins: Air-coupled ultrasonic testing of metals using broadband pulses in through-transmission, Ultrasonic, Vol. 37(1999), p. 19-22

[6] David N. Alleyne and Peter Cawley: The Interaction of Lamb Waves with Defect, IEEE Trans. Ultrason. Ferroelec. Freq. 
KIM et al., Assessment of Wall-Thinning in Carbon Steel Pipe by Using Laser-Generated Guided Wave

Contr., 39A(3) : 381-397, 1992

[7] Kin HM, Lee TH and Jhang KY.: Non-Contact Guided Wave Technique with Enhanced Mode-Selectivity, J Korean Soc NDT, 26(6) : 597-602, 2004.
[8] David A. Hutchins, William M. D. Wright and Gordon Hayward: Air-Coupled Piezoelectric Detection of LaserGenerated Ultrasound, IEEE Trans. Ultrasonic. Ferroelec. Freq. Contr., 41A(6) : 796-805, 1994. 